\title{
Tinnitus and Pediatric Pseudotumor Cerebri Syndrome
}

\author{
Dominique De Vivo ${ }^{1}$ Emanuele David ${ }^{2,3}$ Anna Claudia Romeo ${ }^{1}$ Antonino Costa ${ }^{1}$ Piero Dotto ${ }^{4}$ \\ Rosa Morabito $^{2}$ Giovanni Stroscio ${ }^{2}$ Enrico Maria Mormina ${ }^{2}$ Francesca Granata ${ }^{2}$ Salvatore Savasta ${ }^{5}$ \\ ${ }^{1}$ Department of Pediatrics, University of Messina, Messina, Italy \\ 2 Department of Radiology, University of Messina, Messina, Italy \\ ${ }^{3}$ Department of Radiology, Anatomopathology and Oncology, \\ Sapienza University of Rome, Italy \\ ${ }^{4}$ Faculty of Medicine and Surgery, University of Messina, Messina, Italy \\ ${ }^{5}$ Department of Pediatrics, University of Pavia, IRCCS San Matteo, \\ Pavia, Italy \\ J Pediatr Neurol 2015;13:38-41. \\ Address for correspondence Dominique De Vivo, MD, Department of \\ Pediatrics, University of Messina, Via Consolare Valeria 1, 98125 \\ Messina, Italy (e-mail: dominique.devivo@gmail.com).
}

\author{
Abstract

\section{Keywords} \\ - pseudotumor cerebri \\ syndrome \\ - idiopathic intracranial \\ hypertension \\ - tinnitus \\ - children
}

Pseudotumor cerebri syndrome (PTCS) is defined by increased pressure of cerebrospinal fluid (CSF), with normal CSF contents and without any intracranial disease found on brain imaging. PTCS is a disease with a predilection for childbearing obese women, but it may also occur in children and in man. The most common symptoms include headache, double vision, transient visual obscuration, and pulsatile tinnitus. The reason for which patients with increased CSF pressure experience tinnitus is not clear, and only a few studies have focused on the etiology of this peculiar clinical feature in the context of PTCS presentation. Besides tinnitus, additional otologic manifestations in children with PTCS include aural fullness, low-frequency hearing loss, and vertigo; these symptoms altogether can easily mimic Ménière disease. We hereby present two girls, who presented tinnitus as the first clinical symptom of PTCS, prior to developing headache and visual anomalies, and speculate on a shared pathophysiologic basis for both PTCS and Ménière disease.

\section{Introduction}

Pseudotumor cerebri syndrome (PTCS) is characterized by increased intracranial pressure in the absence of any identifiable anomaly (e.g., hydrocephalus, mass) on brain imaging, and of any concomitant abnormality in cerebrospinal fluid (CSF) contents. ${ }^{1}$ This syndrome typically occurs in obese, postpubertal women, but it may occur even in prepubertal children and in men, who may not be overweight. ${ }^{2,3}$ Idiopathic intracranial hypertension is considered the primary form of PTCS; secondary PTCS is related to a wide array of risk factors and/or comorbidities, such as thrombotic disorders, endocrine diseases, and renal as well as rheumatic illnesses. ${ }^{4-7}$

received

December 22, 2014 accepted after revision February 14, 2015
Issue Theme Pediatric Pseudotumor Cerebri Syndrome; Guest Editors: Vincenzo Salpietro, MD,

Martino Ruggieri, PhD, and Conrad E. Johanson, PhD
Most children with PTCS present with headache and visual defects (e.g., double vision, vision loss) as well as pulsatile tinnitus sometimes associated with additional otologic manifestations. ${ }^{5}$ In fact, PTCS patients can present to otolaryngologists with tinnitus, aural fullness, low-frequency hearing loss, and vertigo; these symptoms altogether can be confused with Ménière disease (MD). ${ }^{8}$ In addition, this raised increased intracranial pressure syndrome can cause spontaneous leakage of CSF from the nose and/or from the ear. ${ }^{8}$ Patients with CSF rhinorrhea and/or otorrhea need a lumber puncture pressure analysis to exclude an underlying PTCS. We hereby report two female prepubertal patients who presented tinnitus as a symptom of primary PTCS, and consider a potential shared pathophysiologic basis for PTCS and MD.

Copyright $\odot 2015$ Georg Thieme Verlag KG Stuttgart · New York

DOI http://dx.doi.org/ $10.1055 / \mathrm{s}-0035-1555152$ ISSN 1304-2580. 


\section{Case Reports}

Over the past few years, we have followed up, at our institution, two female patients who initially presented tinnitus and later developed typical clinical presentation of PTCS (e.g., headache, double vision).

\section{Case 1}

The first patient (case 1) was initially admitted to Messina University hospital at 8 years of age, because of persistently severe tinnitus for 1 month. During her stay in our inpatient area, a grade III bilateral papilledema was noted during diagnostic workup at the funduscopic examination. Magnetic resonance imaging (MRI) showed widening of the optic nerve sheath and bilateral optic papilla protrusion with posterior globe flattening (-Fig. 1A-C). On day 9 after admission, she developed severe headache; we thus performed lumbar puncture. The opening CSF pressure was elevated to $+270 \mathrm{~mm} \mathrm{H}_{2} \mathrm{O}$. She was diagnosed with PTCS and put on acetazolamide (20 mg/kg/day) for 3 months, with good clinical response. After 5 months from the start of therapy, the findings for her on MRI improved ( - Fig. 1D, E). However, the girl continued to complain of persistently moderate to severe episodes of tinnitus during the 12-month follow-up.

\section{Case 2}

The second girl (case 2) was initially admitted to our outpatient area at 9 years of age because of severe tinnitus. Three weeks after the onset of tinnitus, she started to complain of double vision and headache. She was mildly overweight (body mass index $>75$ th percentile), but her neurologic examination was fully normal. The funduscopic examination showed a bilateral papilledema (-Fig. 2) and the brain MRI revealed a transverse sinus stenosis. We performed lumbar puncture to assess CSF opening pressure, which was found to be elevated to $320 \mathrm{~mm}$ $\mathrm{H}_{2} \mathrm{O}$; this confirmed the diagnosis of PTCS. She was then started on acetazolamide $(20 \mathrm{mg} / \mathrm{kg} /$ day $)$ with a good clinical response, and partial reversion of headache and visual disturbances; however, she continued to complain about episodes of tinnitus during the 10-month follow-up.

\section{Discussion}

Pulsatile tinnitus, also known as pulse-synchronous tinnitus, is another common symptom of PTCS, occurring in approximately half of the patients. ${ }^{5}$ Tinnitus is described as the perception of a sound within the ear (e.g., ringing of the ears) when no external sounds are present. Tinnitus is usually pulsatile and may be unilateral or bilateral; in rare occasions it has been

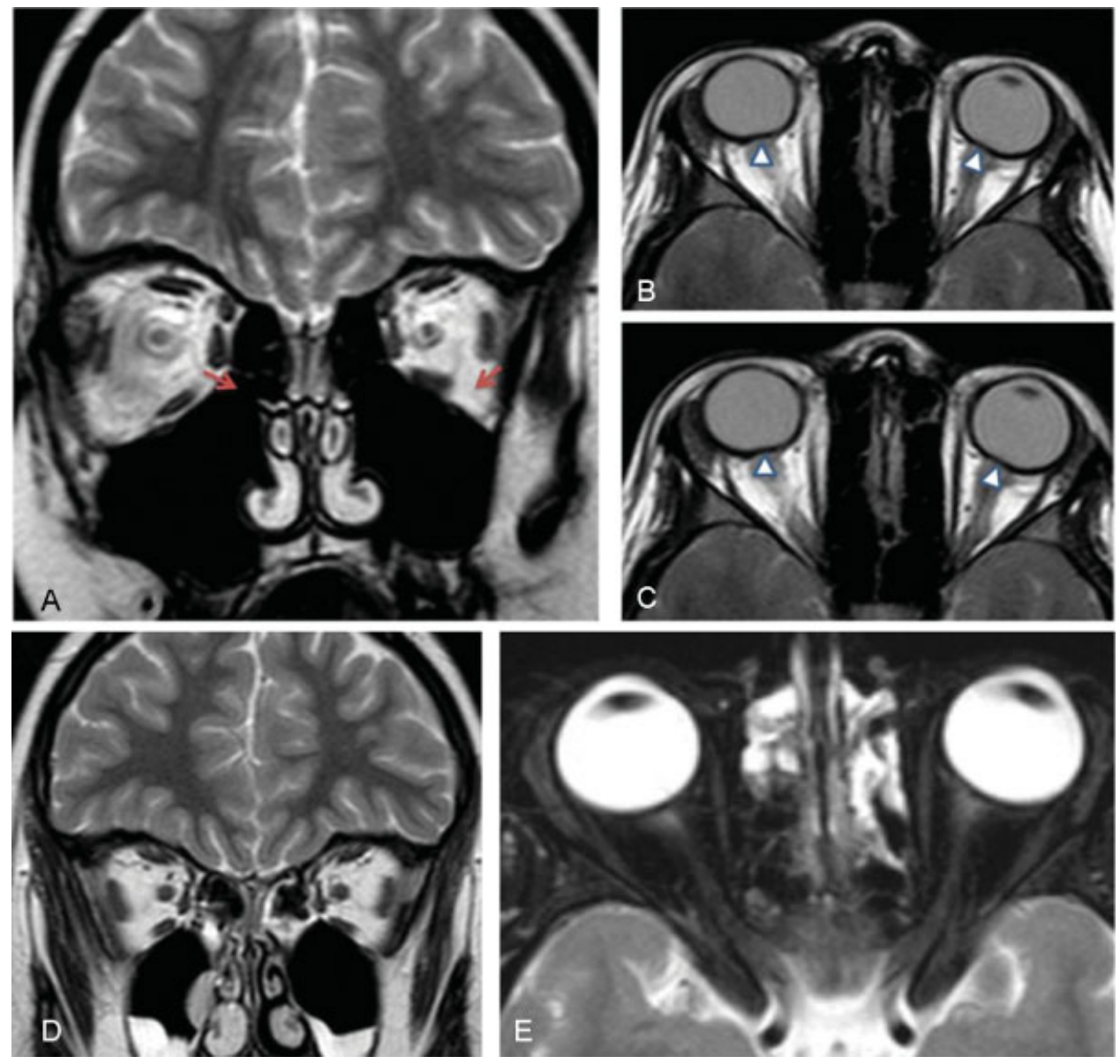

Fig. 1 (A) Coronal fast-spin echo T2-weighted image. (B, C) Axial fast-spin echo T2-weighted images. Bilateral widening (red arrows) of the optic nerve sheath. Bilateral optic papilla protrusion with posterior globe flattening (white arrowheads). (D) Coronal fast-spin echo T2-weighted image. (E) Axial spectral presaturation with inversion recovery image shows the restoration of optic nerve sheath thickness and a normal insertion of the papilla during the follow-up radiologic control. 

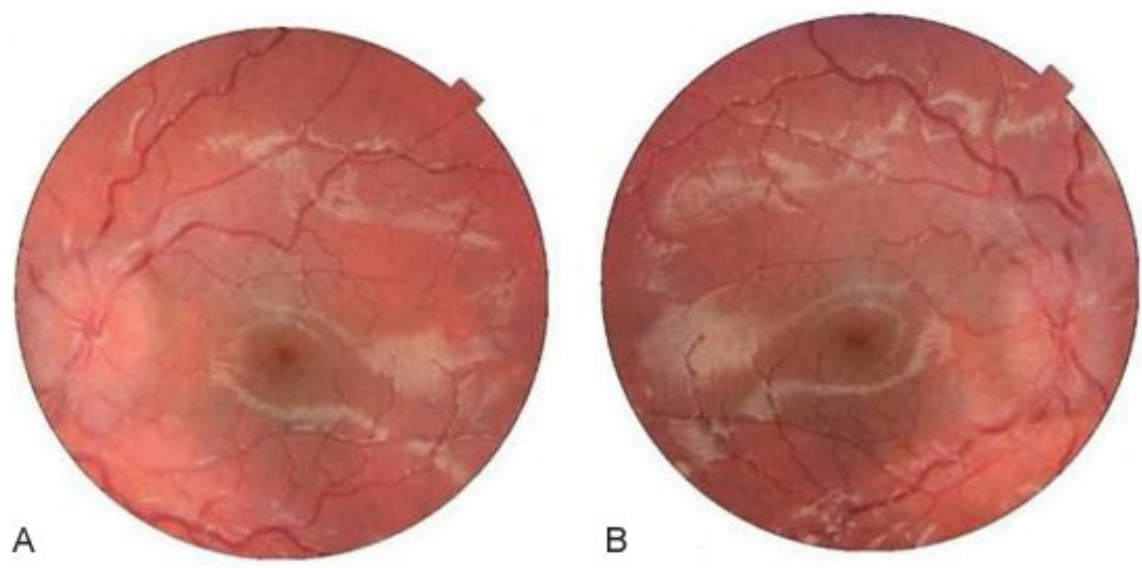

Fig. 2 Papilledema in the right (A) and left (B) eye at the funduscopic examination.

associated with the risk of sensorineural hearing impairment. ${ }^{7,9}$ It has been postulated that tinnitus in PTCS results from a compression of the venous sinuses because of the rise of CSF pressure. This compression may create a turbulent flow in cerebral and (later) inner ear circulation, likely leading to the sensation of tinnitus. ${ }^{9}$ Similarly, to the risk of visual loss due to optic nerve compression, some authors have suggested that neuro-otologic manifestations in PTCS are due to a compression of the cochlea-vestibular nerve. ${ }^{10,11}$

According to this proposed mechanism, the increased CSF pressure constricts the cochlea-vestibular nerve, leading to tinnitus, dizziness, and sensorineural hearing impairment in the context of PTCS.9,10 The abnormal auditory brainstem responses (increased inter-peak latencies), documented in some patients with intracranial hypertension and tinnitus, bring evidence for this assumption. ${ }^{9}$ Notably, the neurootologic manifestations frequently reported in the context of PTCS (i.e., tinnitus, dizziness and sensorineural hearing impairment) may easily mimic MD. ${ }^{7}$ Similarly to PTCS, MD is a clinical entity of still unclear pathophysiology characterized, from a physiologic point of view, by endolymphatic hydrops in the inner ear. ${ }^{7,12}$

Even though the mechanisms underlying the overaccumulation of endolymph in MD are largely unclear, most authors have suggested that a derangement of water homeostasis in the inner ear is a key factor in the initiation and progression of the disease. ${ }^{12}$ To explain the pathophysiology of endolymphatic hydrops, the research in this field has particularly focused in recent years on the disturbed regulation of endolymph flow by the vasopressin-aquaporin-2 system. According to this perspective, endolymphatic hydrops represents the result of alterations in the vasopressin-aquaporin-2 system, leading to a disruption of inner ear fluid homeostasis. ${ }^{12}$

Notably, besides the functioning of the vasopressin-aquaporin channels, the sodium-potassium-adenosine triphosphatase (Na-K-ATPase) system has been identified at the basolateral surface of vestibular dark cells of the inner ear. ${ }^{13}$ Although the role of Na-K-ATPase in regulating endolymph flow awaits elucidation, it has been documented that various hormones (e.g., glucocorticoids, mineral corticoid) are able to stimulate the expression and activity of these ATPase units, likely leading to accumulation of endolymph and MD. ${ }^{12,13}$ The potential involvement of Na-K-ATPase in regulating endolymph flow is supported by many studies that have focused on the central role of salt and water homeostasis in the pathophysiology of MD. This is also indirectly supported by the effectiveness of strict avoidance of salt in the diet of MD patients. ${ }^{12}$ Interestingly, the PTCS patients should also limit their salt intake according to several authors. ${ }^{2} \mathrm{~A}$ feasible explanation is that the ingestion of salt, known to alter the circulating renin and aldosterone, likely activates the $\mathrm{Na}-\mathrm{K}-\mathrm{ATPa}$ e in both the inner ear (endolymph formation) and choroid plexus (CSF production). Indeed, the $\mathrm{Na}-\mathrm{K}-$ ATPase of the choroid plexus has recently been proposed to have a central role in the pathophysiology of PTCS. ${ }^{4} \mathrm{Na}-$ stimulated channels and receptors, in response to hormonal and homeostatic changes (e.g., hyperaldosteronism, hypercortisolism), may augment the flux of $\mathrm{Na}$ into the choroid plexus and nearby cerebral ventricles, ultimately increasing the CSF pressure. ${ }^{4,14}$

Such information on endolymph and CSF contributes new insights on the possible involvement of Na-K-ATPase activity in both MD and PTCS. Altogether, this suggests that otologic and neurologic effects may stem from a background of potentially similar homeostatic derangements. However, further studies need to be performed to explain properly the high occurrence of tinnitus and other otologic disturbances (e.g., hearing loss, vertigo) in children affected by PTCS. This approach will better inform on a possible common pathophysiology of these often associated neuro-otologic manifestations.

\section{References}

1 Friedman DI, Liu GT, Digre KB. Revised diagnostic criteria for the pseudotumor cerebri syndrome in adults and children. Neurology 2013;81(13):1159-1165

2 Khan MU, Khalid H, Salpietro V, Weber KT. Idiopathic intracranial hypertension associated with either primary or secondary aldosteronism. Am J Med Sci 2013;346(3):194-198 
3 Salpietro V, Chimenz R, Arrigo T, Ruggieri M. Pediatric idiopathic intracranial hypertension and extreme childhood obesity: a role for weight gain. J Pediatr 2013;162(5):1084

4 Salpietro V, Polizzi A, Di Rosa G, et al. Adrenal disorders and the paediatric brain: pathophysiological considerations and clinical implications. Int J Endocrinol 2014;2014:282489

5 Rogers DL. A review of pediatric idiopathic intracranial hypertension. Pediatr Clin North Am 2014;61(3):579-590

6 Salpietro V, Ruggieri M, Sancetta F, et al. New insights on the relationship between pseudotumor cerebri and secondary hyperaldosteronism in children. J Hypertens 2012;30(3):629-630

7 Salpietro V, Ruggieri M. Pseudotumor cerebri pathophysiology: the likely role of aldosterone. Headache 2014;54(7):1229

8 Jindal M, Hiam L, Raman A, Rejali D. Idiopathic intracranial hypertension in otolaryngology. Eur Arch Otorhinolaryngol 2009;266(6):803-806
9 Sismanis A, Callari RH, Slomka WS, Butts FM. Auditory-evoked responses in benign intracranial hypertension syndrome. Laryngoscope 1990;100(11):1152-1155

10 Kapoor KG. Etiology of dizziness, tinnitus, and nausea in idiopathic intracranial hypertension. Med Hypotheses 2008;71(2):310-311

11 Møller AR. The role of neural plasticity in tinnitus. Prog Brain Res 2007;166:37-45

12 Seemungal BM, Gresty MA, Bronstein AM. The endocrine system, vertigo and balance. Curr Opin Neurol 2001;14(1):27-34

13 Akiyama K, Miyashita T, Matsubara A, et al. Expression and localization of 11beta-hydroxysteroid dehydrogenase (11betaHSD) in the rat endolymphatic sac. Acta Otolaryngol 2010; 130(2):228-232

14 Salpietro V, Polizzi A, Bertè LF, et al. Idiopathic intracranial hypertension: a unifying neuroendocrine hypothesis through the adrenal-brain axis. Neuroendocrinol Lett 2012;33(6):569-573 